Textures and Microstructures, 1987, Vol. 7, pp. 131-147

Photocopying permitted by license only

(C) 1987 Gordon and Breach Science Publishers Inc.

Printed in the United Kingdom

\title{
Crystallographical Calculation of Earing in Deep Drawing under Various Conditions
}

\author{
NAOYUKI KANETAKE and YASUHISA TOZAWA \\ Department of Iron and Steel Engineering, Nagoya University, Furocho, \\ Chikusaku, 464 Nagoya, Japan
}

(Received 20 October 1986; in revised form 3 December 1986)

Earing in the cup drawing of sheet metals is calculated quantitatively using a crystallographical theory with measured texture data. In the analysis a polycrystalline sheet is simplified to be an aggregate of many single crystals with various orientations, and a crystallite orientation distribution function which is calculated from the measured texture is used as a volume fraction of a certain oriented crystal. The circumferential distribution of radial strain in a flange of a blank being drawn is calculated by considering a restricted glide in a crystal, and then cup height at each peripheral position of a drawn cup is calculated from this distribution of radial strain. For an aluminium, a copper and two steel sheets the calculated cup profiles are compared with experimental ones under various drawing conditions of dimension of a punch, a die and a blank. The result shows that earing in the drawn cup can be predicted satisfactorily by the present calculation for a wide range of drawing conditions and materials.

KEY WORDS: Earing, polycrystalline sheet, drawing conditions.

\section{INTRODUCTION}

There are many problems lying on an interdisciplinary research field and making them clear becomes very important nowadays. A problem lying on the boundary between the field of materials science and the field of sheet metal forming technology is treated in the present work. In the former field many researches are made energetically to clear quantitatively deformation behaviour of a polycrystalline material based on detailed deformation mode of a single crystal. However the macroscopic phenomena which can be made clear by them are only simple behaviours of isotropic materials under a uniaxial stress state at most. These results can't be used directly to solve technological problems. In the latter field, on 
the other hand, most of the problems are tried to be solved by use of a continuous theory, but an anisotropic behaviour of a sheet metal is very difficult to make clear by the theory. Although the behaviour is well known in the qualitative relation with behaviour of crystals, clearing the quantitative relation is a future subject. The authors have attempted to calculate quantitatively macroscopic phenomena in a deformation of sheet metals by use of a crystallographical theory (Kanetake, Tozawa and Kato, 1981, Kanetake, Tozawa and Otani, 1983 and Kanetake, Tozawa and Yamamoto, 1985). In these works a polycrystal is assumed as an aggregate of many single crystals with a simple deformation mode, and measured texture data represented by a three-dimensional crystallite orientation distribution function is used with the crystallographical theory. The analyzing method was shown to be very useful for predicting quantitatively anisotropic behaviour of the sheet metals in practice use.

In the present paper the analyzing method is applied to predict earing in a cup drawn under various drawing conditions for various textured sheets. On the earing, in the materials science field, the relationship between earing pattern and texture type has been found empirically, and the earing of a single crystal sheet has been predicted by use of a crystallographical theory (Tucker, 1961 and Vieth and Whiteley, 1964). In the sheet metal forming field, on the other hand, the relationship between earing pattern and planar anisotropy of an $r$-value has been found empirically, and calculating the earing by use of a slip-line field theory or a finite element method has been carried out by some researchers. Through those many previous researches, the earing pattern of a drawn cup is predictable qualitatively from the texture or mechanical properties of a sheet blank, but is not quantitatively. The distribution of cup height along a circumference of the drawn cup is calculated and compared with experimental ones in the present work. From the results, it is confirmed that the analyzing method is useful for a wide range of drawing conditions and materials used in the practice.

\section{THEORETICAL PREDICTIONS}

\section{Assumptions used in the analysis}

(1) At any stage during a deep drawing operation there is a tensile stress $(\sigma)$ radially, a compressive stress $(-\sigma)$ circumferentially and 
zero stress normal to the sheet surface at any point in a flange of the blank.

(2) As a deformation mode of a single crystal, only a restricted glide on following slip systems is assumed:

a) 12 slip systems of $\{111\}\langle 110\rangle$ for a FCC single crystal

b) 48 slip systems of $\{110\}\langle 111\rangle,\{112\}\langle 111\rangle$ and $\{123\}\langle 111\rangle$ for a BCC single crystal.

(3) On the all slip systems a relationship between the shear stress, $\tau$, and the shear strain, $\gamma$, is expressed by a following equation,

$$
\tau=\mu \sigma=k \gamma^{n}+\tau_{0}
$$

where $\mu$ is the Schmid factor and $k, n$ and $\tau_{0}$ are constants.

(4) When more than one slip system operate simultaneously, the macroscopic strain is taken as a sum of its components calculated for each system separately.

(5) A textured polycrystalline sheet metal is simplified as an aggregate of many crystals with various orientations whose volume fractions are expressed by means of a crystallite orientation distribution function.

(6) The change in orientation of each grain, i.e. change of the texture, which occurs during the practical drawing, is ignored in resolved shear stress, but is taken into account in resolving strain.

\section{Calculation method}

Earing is attributed to an inhomogeneity of radial strain along the circumferential direction in a flange of a circular blank. Therefore, the circumferential distribution of radial strain during cup drawing is calculated, and then, using this result, the circumferential distribution of cup height is calculated.

First, consider the drawing of a circular single crystal blank with an orientation represented by the Euler angles $\psi, \theta$ and $\phi$, which relate crystal axes ([100], [010], [001]) to sheet reference axes (rolling-, transverse- and normal-direction: RD, TD, ND). At any point in the flange of the blank, the directions of principal stresses are radial, circumferential and normal directions $\left(S_{1}, S_{2}, S_{3}\right)$, and these directions are expressed by a parameter $\alpha$ which is an angle to the rolling direction of a polycrystalline sheet, Figure 1 . If the 


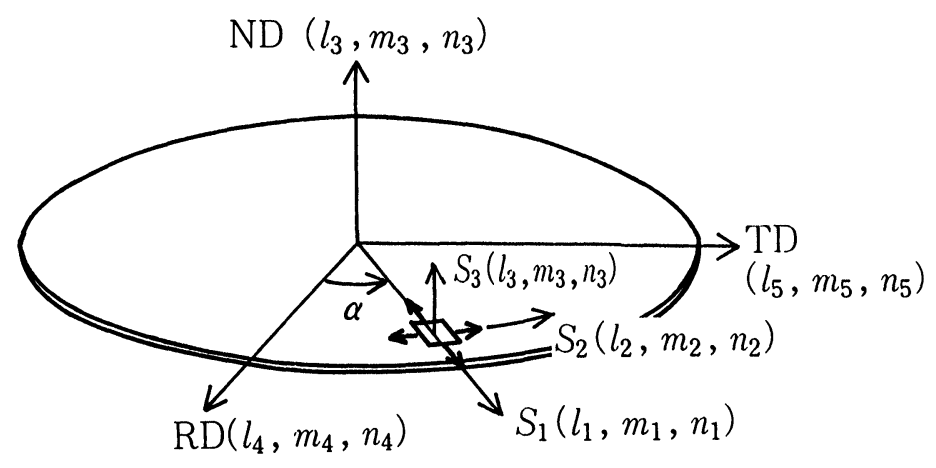

Figure 1 Diagram illustrating sheet reference axes (RD, TD, ND) and principal stress axes $\left(S_{1}, S_{2}, S_{3}\right)$.

direction cosines of $S_{1}, S_{2}, S_{3}, R D, T D$ and $N D\left(S_{3}\right)$, with respect to the crystal axes, are defined by $\left(l_{1}, m_{1}, n_{1}\right),\left(l_{2}, m_{2}, n_{2}\right)$, $\left(l_{3}, m_{3}, n_{3}\right),\left(l_{4}, m_{4}, n_{4}\right),\left(l_{5}, m_{5}, n_{5}\right)$ and $\left(l_{3}, m_{3}, n_{3}\right)$ respectively, they can be expressed in terms of the four angles $\psi, \theta, \phi$ and $\alpha$ as follows:

$$
\begin{aligned}
l_{3} & =-\sin \theta \cos \phi, \quad m_{3}=\sin \theta \sin \phi, \quad n_{3}=\cos \theta \\
l_{4} & =\cos \psi \cos \theta \cos \phi-\sin \psi \sin \phi \\
m_{4} & =-\cos \psi \cos \theta \sin \phi-\sin \psi \cos \phi \\
n_{4} & =\cos \psi \sin \theta \\
l_{5} & =n_{3} m_{4}-m_{3} n_{4}, \quad m_{5}=l_{3} n_{4}-n_{3} l_{4}, \quad n_{5}=m_{3} l_{4}-l_{3} m_{4} \\
l_{1} & =l_{5} \cos \alpha-l_{4} \cos \alpha, \quad m_{1}=m_{5} \cos \alpha-m_{4} \sin \alpha \\
n_{1} & =n_{5} \cos \alpha-n_{4} \sin \alpha, \quad l_{2}=l_{4} \cos \alpha+l_{5} \sin \alpha \\
m_{2} & =m_{4} \cos \alpha+m_{5} \sin \alpha, \quad n_{2}=n_{4} \cos \alpha+n_{5} \sin \alpha
\end{aligned}
$$

On the other hand, for a given slip system $N:\left(h_{N} k_{N} l_{N}\right)\left[u_{N} v_{N} w_{N}\right]$ from the set of all considered slip systems $(N=1,2, \cdots)$, if the direction cosines of the slip plane $\left(h_{N} k_{N} l_{N}\right)$ and the slip direction $\left[u_{N} v_{N} w_{N}\right]$, with respect to the principal stress axes $\left(\mathrm{S}_{1}, \mathrm{~S}_{2}, \mathrm{~S}_{3}\right)$, are $\left(a_{N}, b_{N}, c_{N}\right)$ and $\left(d_{N}, e_{N}, f_{N}\right)$ respectively, they are expressed as 
follows:

$$
\begin{aligned}
& a_{N}=\left(h_{N} l_{1}+k_{N} m_{1}+l_{N} n_{1}\right) /\left(h_{N}^{2}+k_{N}^{2}+l_{N}^{2}\right)^{1 / 2} \\
& b_{N}=\left(h_{N} l_{2}+k_{N} m_{2}+l_{N} n_{2}\right) /\left(h_{N}^{2}+k_{N}^{2}+l_{N}^{2}\right)^{1 / 2} \\
& c_{N}=\left(h_{N} l_{3}+k_{N} m_{3}+l_{N} n_{3}\right) /\left(h_{N}^{2}+k_{N}^{2}+l_{N}^{2}\right)^{1 / 2} \\
& d_{N}=\left(u_{N} l_{1}+v_{N} m_{1}+w_{N} n_{1}\right) /\left(u_{N}^{2}+v_{N}^{2}+w_{N}^{2}\right)^{1 / 2} \\
& e_{N}=\left(u_{N} l_{2}+v_{N} m_{2}+w_{N} n_{2}\right) /\left(u_{N}^{2}+v_{N}^{2}+w_{N}^{2}\right)^{1 / 2} \\
& f_{N}=\left(u_{N} l_{3}+v_{N} m_{3}+w_{N} n_{3}\right) /\left(u_{N}^{2}+v_{N}^{2}+w_{N}^{2}\right)^{1 / 2}
\end{aligned}
$$

The shear strain, $\gamma_{N}$, generated by the shear stress, $\tau_{N}$, in the slip system $N$ is calculated as follows using equations (1), (2) and (3).

$$
\begin{array}{ll}
\gamma_{N}=\left[\left\{\sigma\left(a_{N} d_{N}-b_{N} e_{N}\right)-\tau_{0}\right\} / k\right]^{1 / n} & \left(\tau_{N}>\tau_{0}\right) \\
\gamma_{N}=0 & \left(\tau_{N}<\tau_{0}\right)
\end{array}
$$

Then, the radial strain at a given $\alpha$ in the flange of the single crystal blank, $\varepsilon_{s}(\psi, \theta, \phi)$, can be calculated as follows.

$$
\varepsilon_{s}(\psi, \theta, \phi)=K \sum_{N}\left[\left\{\left(a_{N} d_{N}-b_{N} e_{N}\right)-\mu_{0}\right\}^{1 / n}\left|b_{N}\right|\right]
$$

where $K=(\sigma / K)^{1 / n}, \mu_{0}=\tau_{0} / \sigma$ and $\sum_{N}$ expresses a sum over all the slip systems considered.

For the textured polycrystalline sheet, at the first step its texture is measured and represented by an orientation distribution function, $w(\psi, \theta, \phi)$. Then $\varepsilon_{s}(\psi, \theta, \phi)$ calculated by Eq. (5) for each single crystallite orientation is weighted by $w(\psi, \theta, \phi)$ and averaged over all orientations, i.e. the radial strain at a given $\alpha$ in the flange of the polycrystalline circular blank, $\varepsilon_{p}$, is calculated as follows:

$$
\varepsilon_{p}=\frac{1}{8 \pi^{2}} \iiint \varepsilon_{s}(\psi, \theta, \phi) w(\psi, \theta, \phi) \sin \theta d \psi d \theta d \phi
$$

Using the $\varepsilon_{p}$, the cup height at each point along a circumference, $h$, is calculated as follows.

$$
\begin{aligned}
h & =\left(h_{w} / \bar{\varepsilon}_{p}\right) \cdot \varepsilon_{p}+r_{p}+t_{0} \\
h_{w} & =\left(R_{b}^{2}-R^{2}\right) /\left(R_{p}+R_{d}\right) \\
R^{2} & =2\left(r_{p}+t_{0} / 2\right)^{2}+\left(r_{p}+t_{0} / 2\right)\left(R_{p}-r_{p}\right) \pi+\left(R_{p}-r_{p}\right)^{2}
\end{aligned}
$$

where $R_{d}, R_{p}, r_{p}, R_{b}$ and $t_{0}$ are dimensions of a die, a punch and a 


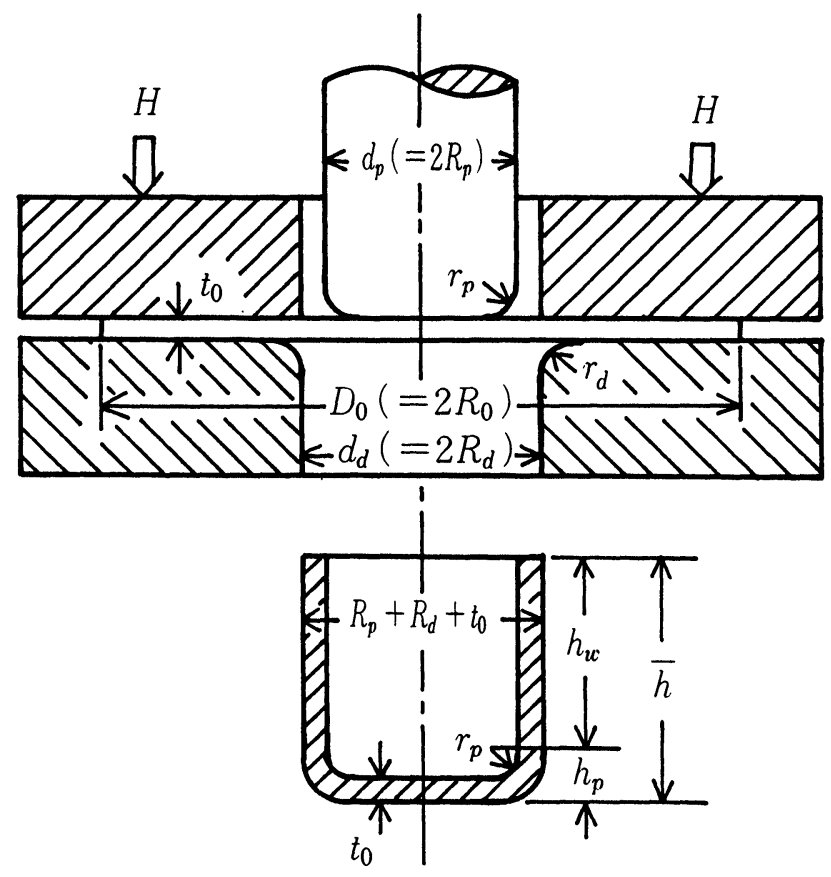

Figure 2 Dimensions of a drawing tool, a blank and a drawn cup.

blank as shown in Figure 2, and $\bar{\varepsilon}_{p}$ is an average value of the $\varepsilon_{p}$ along a circumference.

As described above, by measuring the texture of the sheet to be drawn and giving values of $n$ and $\mu_{0}$, a feature of the earing in the drawn cup, i.e. the distribution of the cup height along a circumference can be calculated under various drawing conditions. In the present work the calculation was made numerically at every $5^{\circ}$ of $\psi$, $\theta, \phi$ and $\alpha$ respectively under $n=1.0$ and $\mu_{0}=0.05$ (Kanetake, Tozawa and Otani, 1983 and Kanetake, Tozawa and Yamamoto, 1985).

\section{EXPERIMENTAL PROCEDURE}

\section{Materials}

As a FCC material, an annealed aluminium sheet and an annealed copper sheet, and as a BCC material, a low carbon steel sheet and a 
Table 1 Mechanical properties of used sheets

\begin{tabular}{|c|c|c|c|c|c|c|c|}
\hline \multirow[t]{2}{*}{ Sheet } & \multirow{2}{*}{$\begin{array}{l}\text { Mean yield } \\
\text { stress, } \\
\mathrm{kg} / \mathrm{mm}^{2}\end{array}$} & \multirow{2}{*}{$\begin{array}{l}\text { Mean tensile } \\
\text { strength, } \\
\mathrm{kg} / \mathrm{mm}^{2}\end{array}$} & \multirow{2}{*}{$\begin{array}{l}\text { Mean uniform } \\
\text { elongation, \% }\end{array}$} & \multirow{2}{*}{$\begin{array}{l}\text { Mean } \\
n \text {-value }\end{array}$} & \multicolumn{3}{|c|}{$r$-value } \\
\hline & & & & & $r_{0}$ & $r_{45}$ & $r_{90}$ \\
\hline Aluminium & 3.7 & 9.4 & 32.7 & 0.272 & 0.64 & 1.31 & 0.67 \\
\hline Copper & 4.2 & 21.4 & 35.5 & 0.504 & 0.85 & 1.45 & 0.56 \\
\hline Low-carbon steel & 14.7 & 30.5 & 28.7 & 0.268 & 1.46 & 1.90 & 2.07 \\
\hline High-strength steel & 41.1 & 58.3 & 17.4 & 0.185 & 1.33 & 0.96 & 1.53 \\
\hline
\end{tabular}

high-strength steel sheet of a precipitation hardening type were used in the experiment. They were all commercially produced sheets and their mechanical properties which were obtained from uniaxial tensile tests in three directions to the rolling direction are given in Table 1. The nominal thickness was $0.8 \mathrm{~mm}$ for all sheets.

\section{Texture measurement}

The average texture over the thickness was measured using the method proposed by Lopata and Kula (1962). A plane of a specimen to be measured and one quadrant of a pole figure to be determined are illustrated in Figure 3. A composite specimen for the texture measurement was prepared using the procedure of Elias and Heckler (1967). Three pole figures for each specimen, (111), (200), (220) for a FCC material and (110), (200), (222) for a BCC

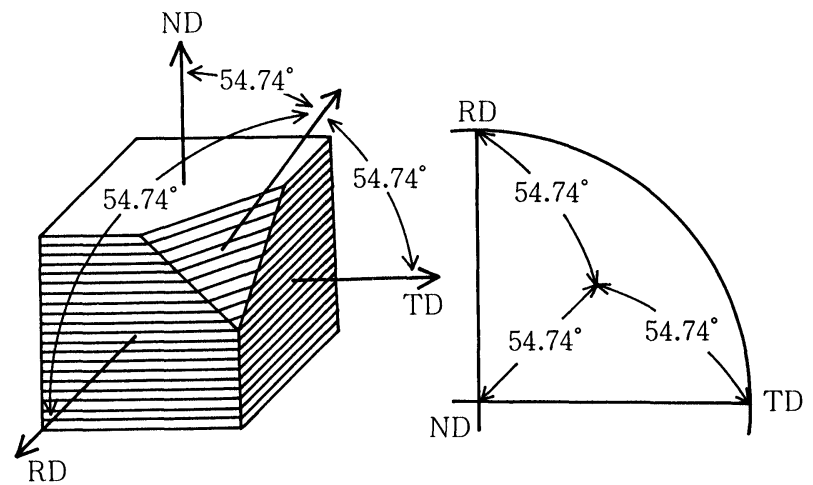

Figure 3 Oblique plane for determining averaged pole figure through the sheet thickness by Schulz reflection method. 
material, were measured, and using those data the orientation distribution function was calculated according to the method of Bunge (1965) or Roe (1965).

\section{Cup drawing}

For each material circular blanks were deep drawn with various dies, punches and blanks whose dimensions are shown in Table 2. Of all the dimensions, a die diameter, $d_{d}$, a punch diameter, $d_{p}$, a punch profile-radius, $r_{p}$, a blank diameter, $D_{0}$, and a blank thickness, $t_{0}$, are used in the theoretical calculation.

Table 2 Cup drawing conditions

\begin{tabular}{|c|c|c|c|c|}
\hline Die & $\begin{array}{l}\text { Diameter } \\
\text { Profile-radius }\end{array}$ & $\begin{array}{l}d_{d} / \mathrm{mm} \\
r_{d} / \mathrm{mm}\end{array}$ & \multicolumn{2}{|c|}{$\begin{array}{c}40 \\
4,10\end{array}$} \\
\hline Punch & $\begin{array}{l}\text { Diameter } \\
\text { Profile-radius }\end{array}$ & $\begin{array}{l}d_{p} / \mathrm{mm} \\
r_{p} / \mathrm{mm}\end{array}$ & $\begin{array}{c}38 \\
4,10,19\end{array}$ & $\begin{array}{l}37 \\
10\end{array}$ \\
\hline Blank & $\begin{array}{l}\text { Diameter } \\
\text { Thickness }\end{array}$ & $\begin{array}{l}D_{0} / \mathrm{mm} \\
t_{0} / \mathrm{mm}\end{array}$ & \multicolumn{2}{|c|}{$\begin{array}{c}1.5 d_{p}, 1.7 d_{p}, 1.9 d_{p}(\mathrm{FCC}) \\
1.4 d_{p}, 1.7 d_{p}, 2.0 d_{p}(\mathrm{BCC}) \\
0.8\end{array}$} \\
\hline \multicolumn{2}{|c|}{ Blank holding force } & & $\begin{array}{l}\text { according to th } \\
\text { of Fukui et al., }\end{array}$ & \\
\hline \multicolumn{2}{|c|}{ Lubricant } & \multicolumn{3}{|c|}{ Rapeseed-oil } \\
\hline \multicolumn{2}{|c|}{ Drawing speed } & \multicolumn{3}{|c|}{ About $20 \mathrm{~mm} / \mathrm{min}$} \\
\hline
\end{tabular}

The height of the drawn cup was measured continuously along a circumference with a dial gauge which has a voltage signal output, and the data were sampled using a microcomputer. After the sampling was finished, two height data at diametrically opposite sites were averaged to exclude error due to eccentricity of the blank to the tool, then the cup profile was drawn on an $X-Y$ plotter.

\section{RESULTS AND DISCUSSION}

Figures 4, 5, 6 and 7 show the crystallite orientation distribution for the four sheets, which were used for calculating the cup profiles. 


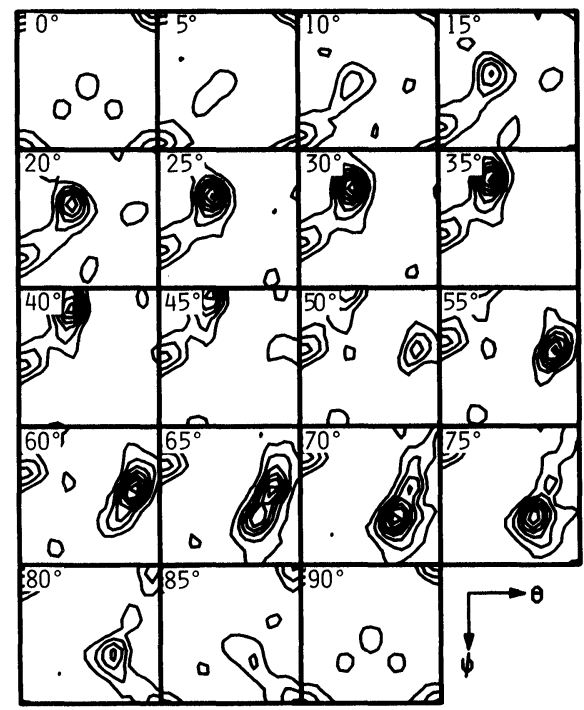

Figure 4 Crystallite orientation distribution of annealed aluminium sheet. Astep of lines shows twice as large as random intensity level.

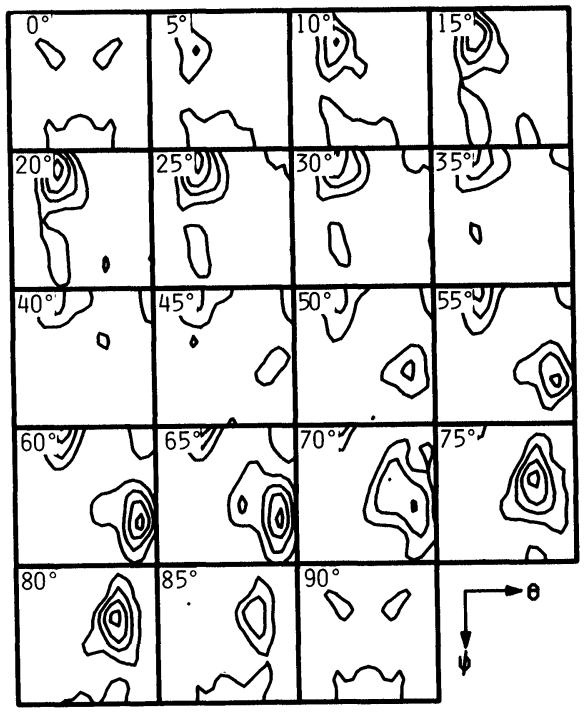

Figure 5 Crystallite orientation distribution of annealed copper sheet. Astep of lines shows twice as large as random intensity level. 


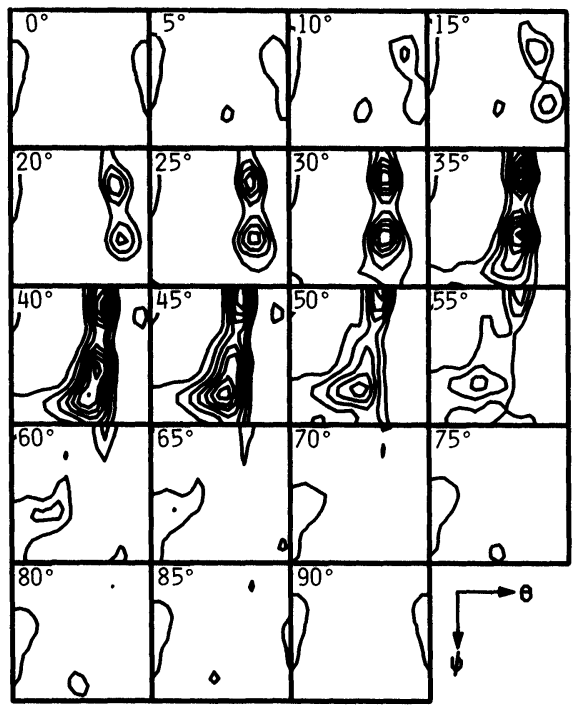

Figure 6 Crystallite orientation distribution of low carbon steel sheet. Astep of lines shows twice as large as random intensity level.

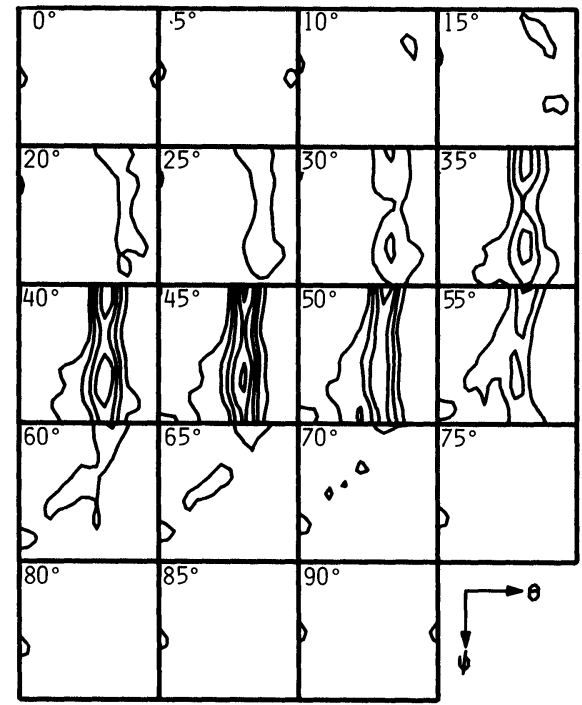

Figure 7 Crystallite orientation distribution of high-strength steel sheet. Astep of lines shows twice as large as random intensity level. 

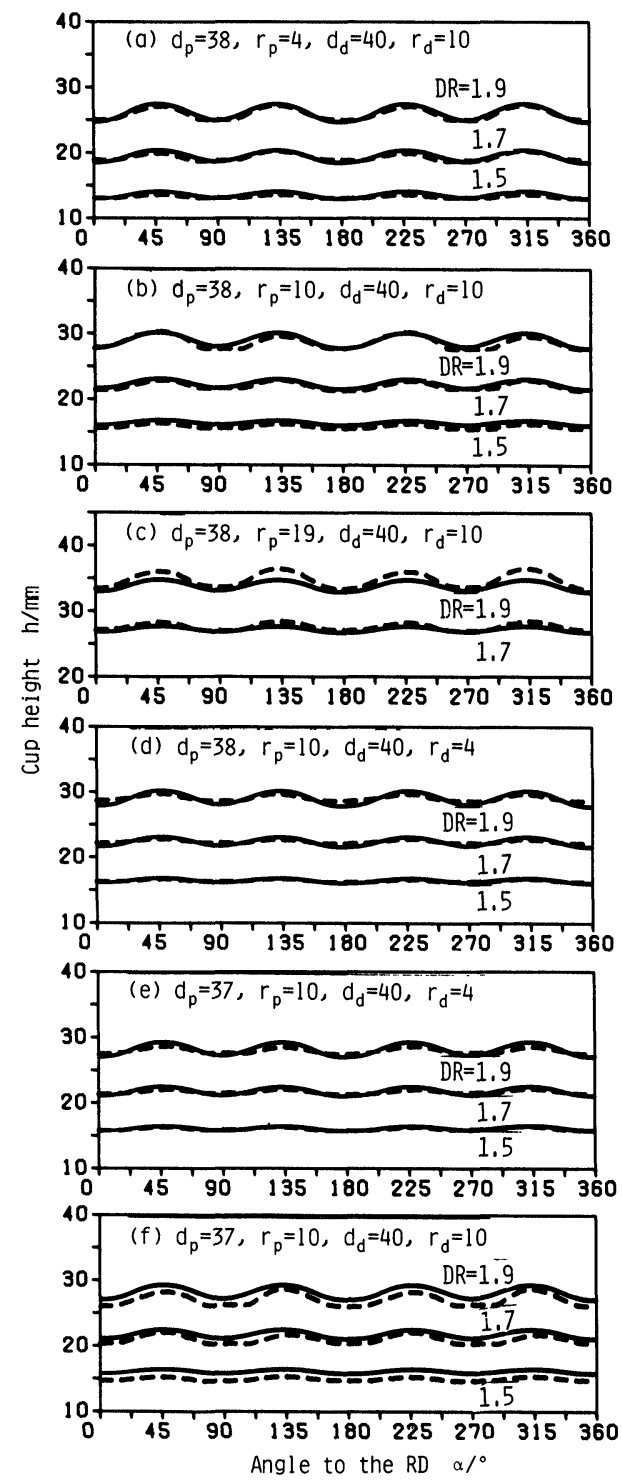

Figure 8 Calculated (solid line) and experimental (dashed line) profiles of cups drawn under various drawing conditions for annealed aluminium sheet. DR: drawing ratio. 
Figures 8, 9, 10 and 11 show the cup profiles calculated under various drawing conditions for the four sheets respectively, and they can be compared with the experimental profiles in those figures. The cups drawn from the aluminium sheet (Figure 8) and the high-strength steel sheet (Figure 11) have both typical four earing, $45^{\circ}$-earing in the aluminium sheet cup and $0^{\circ}-90^{\circ}$-earing in the high-strength steel sheet cup. A detailed observation in the latter can find that the ear at $90^{\circ}$ is wider than that at $0^{\circ}$. The cup of the copper sheet (Figure 9) has also four earing, but a position of the ears is a little out of $45^{\circ}$ and the trough at $0^{\circ}$ is deeper and narrower than that at $90^{\circ}$. The cup of the low carbon steel sheet (Figure 10) has six earing. Although the ears are small on the whole, it's distinct that the ear at $0^{\circ}$ is smaller than the others. In comparing the calculated cup profiles with the experimental ones it can be found, that there is a close agreement between them, in other words, for a principal feature of the experimental cups not only a number of ears but also a detailed position and height of each ear and trough can be predicted by the calculation method shown in the present work. This is a large advantage of the calculation method using the measured texture data for analyzing earing.

In cup drawing with punches which have various profile-radii the calculated and experimental cup profiles agree similarly well for respective radii, though a little difference between both profiles is seen in the cups drawn with a spherical punch (See (a), (b) and (c) in each figure). Reducing of sheet thickness due to stretch deformation at the punch profile and increasing of it near a rim of a cup wall are both not taken into consideration in the present calculation. The effects of both these assumptions, consequently, compensate each other, and good agreement between calculated and experimental cup profiles can be seen under conditions of widely varied punch profile-radius.

The results of cups drawn with different diameter punches are seen in comparing (b) and (e) or (d) and (f) of each figure. Clearances between a punch and a die are $1.25 t_{0}$ and $1.9 t_{0}$ for $d_{p}=38$ and $37 \mathrm{~mm}$ respectively. Although the wall of the cup drawn under a condition of the smaller clearance is ironed slightly at a trough, the effect of ironing scarcely appears on the profile of the cup. In a case of drawing under a condition of the larger clearance, 

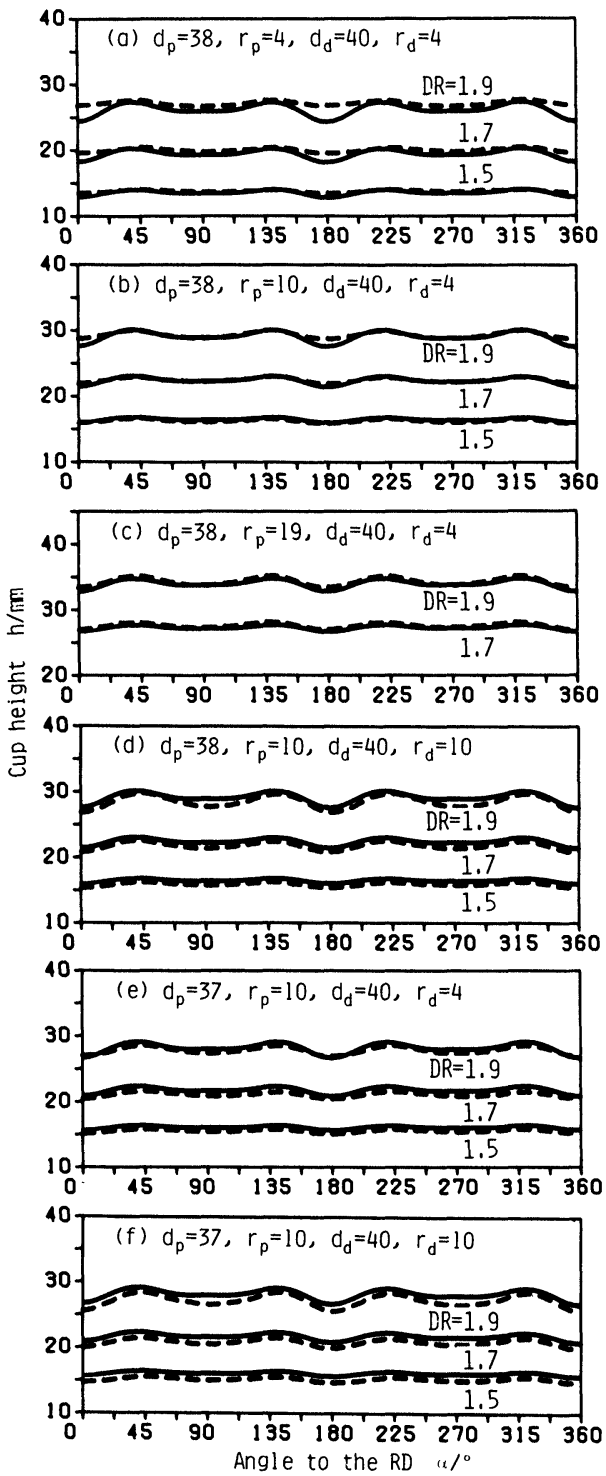

Figure 9 Calculated (solid line) and experimental (dashed line) profiles of cups drawn under various drawing conditions for annealed copper sheet. DR: drawing ratio. 

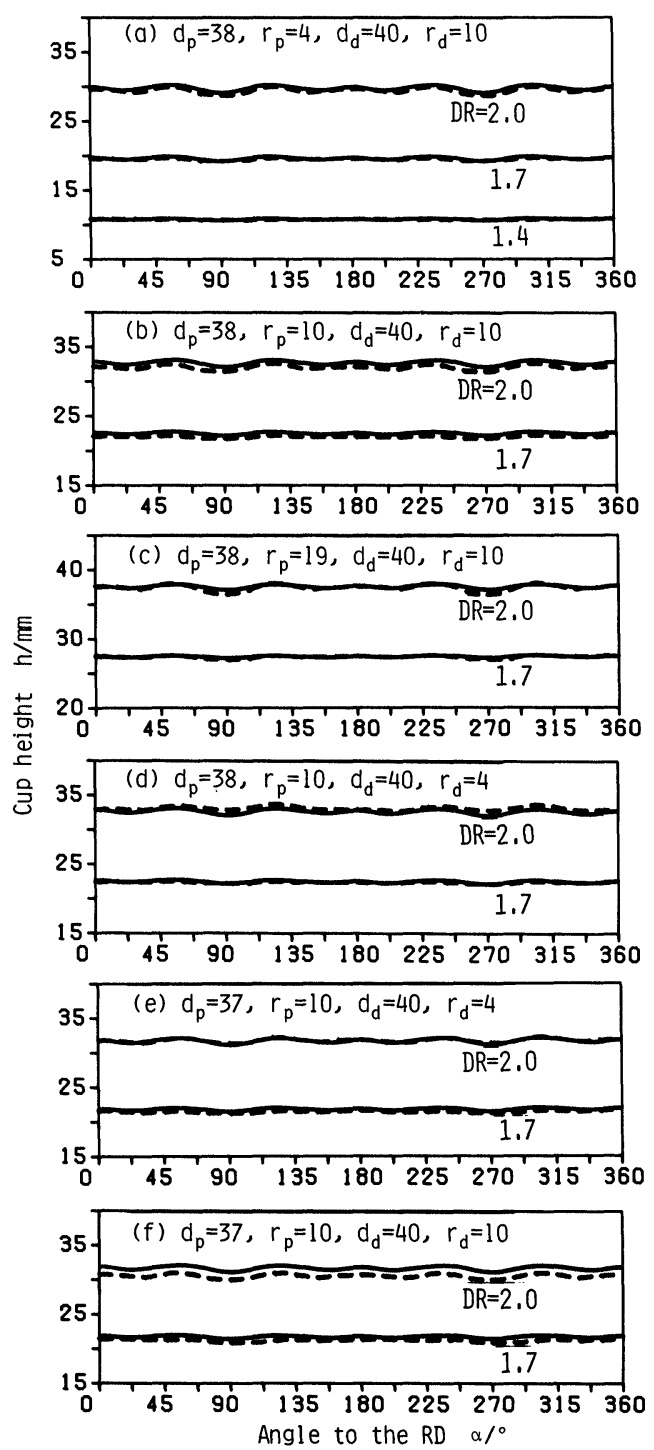

Figure 10 Calculated (solid line) and experimental (dashed line) profiles of cups drawn under various drawing conditions for low carbon steel sheet. DR:drawing ratio. 

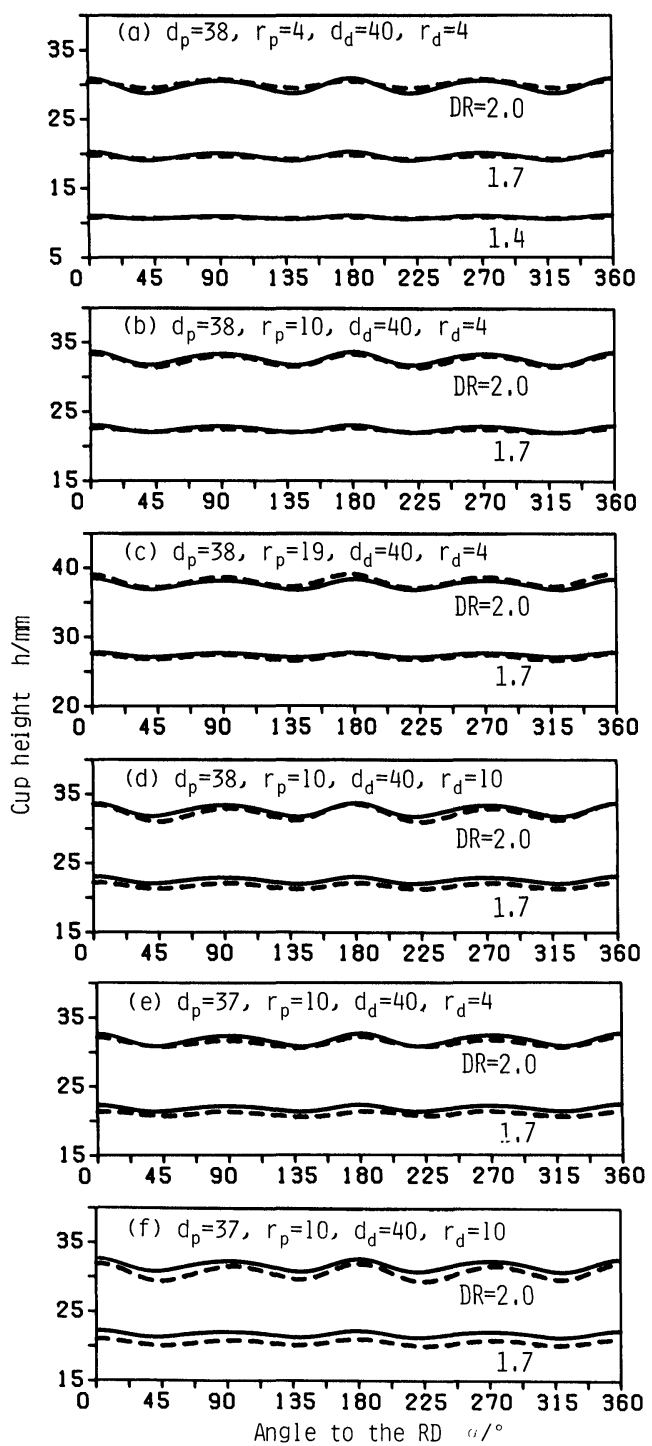

Figure 11 Calculated (solid line) and experimental (dashed line) profiles of cups drawn under various drawing conditions for high-strength steel sheet. DR: drawing ratio. 
the experimental cup height is a little lower than the calculated one. This is due to the fact that the rim of the wall of the cup drawn under the condition is slightly curled because of remaining of bending deformation at a die shoulder. In spite of this fact, the calculated profile can predict very well the experimental one under the condition of a clearance range used in the practice.

The effect of a die profile-radius $r_{d}$ can be also seen in the figures, and $r_{d}=4$ and $10 \mathrm{~mm}$ are equivalent to $5 t_{0}$ and $12.5 t_{0}$ respectively. Although the radii are close to a lower and an upper bound of that used in the practice, the effect of the radii on the cup profile is very small. The fact supports the present calculation method in which the die profile-radius is not taken into consideration.

For a BCC metal, as a deformation mode of a single crystal besides of the restricted glide considered in the present work, a pencil glide along $\langle 111\rangle$ slip direction is observed in general. This problem as well as on the values of $n$ and $\mu_{0}$ was discussed in the previous works (Kanetake, Tozawa and Otani, 1983 and Kanetake, Tozawa and Yamamoto, 1985), and the assumptions used in the present work were decided to be suitable. Although many calculations of prastic properties have been made, up to now, using the Taylor theory or certain modification of the theory, another deformation model presented by Sachs (1928) was modified in the present calculation, which is the model for constant stress and violates the condition of minimum deformation work. Besides this, very bold assumptions are used for analyzing deformation behaviour of a textured polycrystalline sheet, that is, a change of the texture during cup drawing, an effect of a grain boundary or a next grain, an inhomogeneity of a stress state in a flange or of strain along a cup wall and so on are not considered at all in the present calculation. In spite of the fact, very close quantitative agreement between theory and practice was obtained in a wide range of drawing conditions and materials. This implies that considering only glide in each crystal grain separated from other grains is very useful for calculating deformation or strain, that a directional dependency of the deformation is affected significantly by the texture, and that a similar crystallographical calculation using measured texture data is very useful for analyzing not only earing of the drawn cup but also other macroscopic deformation behaviour of an anisotropic material. 


\section{CONCLUSION}

Aluminium, copper and steel sheets were cup drawn under various conditions of geometry of a punch, a die and a blank, and their cup profiles were examined. On the other hand the cup profiles of those sheets were calculated using the crystallographical theory with the measured texture data for the same drawing conditions. As a result of comparing the experimental and calculated cup profiles, it was found that the principal feature of the cup, such as a number, a position and height of the ears can be predicted satisfactorily for a wide range of drawing conditions and materials by the present calculation method. Thus the calculation is very useful for technological purposes, for example a quantitative prediction of earing for various sheets or a computer simulation of a relationship between the texture and the earing. Furthermore, the similar calculation method using texture data is very useful for analyzing other problems lying between the materials science field and the sheet metal forming field.

\section{References}

Bunge, H. J. (1965) Z. Metallkde., 56, 872-874.

Elias, J. A. and Heckler, A. J. (1967) Trans. Met. Soc, AIME, 239, 1237-1241.

Fukui, S., Yoshida, K. and Abe, K. (1954) Rep. Inst. Sci. Tech. Univ. Tokyo, 8, 23-30.

Kanetake, N., Tozawa, Y. and Kato, T. (1981) Proc. 6th ICOTOM, 2, 1101-1110.

Kanetake, N., Tozawa, Y. and Otani, T., (1983) Int. J. Mech. Sci., 25, 337-345.

Kanetake, N., Tozawa, Y. and Yamamoto, S., (1985) Int. J. Mech. Sci., 27, 249-256.

Lopta, S. L. and Kula, E. B. (1962) Trans. Met. Soc. AIME, 224, 865-866.

Roe, R. J. (1965) J. Appl. Phys., 36, 2024-2031.

Sachs, G. (1928) Z:VDI, 72, 734.

Tucker, G. D. G. (1961) Acta Metallugica, 9, 275-280.

Vieth, R. W. and Whiteley, R. L. (1964) Proc. IDDRG Colloq. 